\title{
Arithmetic Operations Of Symmetric Trapezoidal Fuzzy Random Variables
}

\author{
Dr. C. Senthil Murugan \\ Assistant Professor \\ Department of Mathematics, \\ Ponnaiyah Ramajayam Institute of Science and Technology (PRIST) Deemed to be University, \\ Kumbakonam campus, Assur-612 501. \\ Email: senthilmurugan1978@gmail.com
}

\begin{abstract}
In this paper, we deliberate a new term symmetric trapezoidal fuzzy random variable with mean $\mu$ and standard deviation $\sigma$ respectively and that we discuss about the membership functions of arithmetic operations between two symmetric trapezoidal fuzzy random variables, verify through here enclosed with suitable numerical examples.

Keywords - Fuzzy random variable, Trapezoidal fuzzy random variable, arithmetic operations, symmetric trapezoidal fuzzy random variable.
\end{abstract}

\section{INTRODUCTION}

The fuzzy set was introduced independently by Zadeh [8] in the year 1965, he provided a sound theoretical setting for the notion of fuzzy sets, as a class of objects with a continuum of grades of membership function. The membership function is one of the most useful tool for expressing the fuzziness. Also, depending the nature or shape of membership function of a fuzzy number can classified in different ways, such as triangular fuzzy number, trapezoidal fuzzy number etc.

The concept of fuzzy random variable, that extends the classical definition of random variable, was introduced by Feron [1] and developed by some authors like Kwakernaak [2, 3], Puri and Ralescu [4, 5] among others. In this paper, we follow kwakernaak fuzzy random variable because the expected value and variance are fuzzy. Rajan. D and Senthil murugan. C [6] derived the concept triangular fuzzy random variable from the normal distribution and proved some stochastic comparison such as likelihood, hazard and stochastic orders.

In this manner, we derive the trapezoidal fuzzy random variable from the normal distribution, so that it satisfies all characteristics and properties of normal distribution as well as the triangular fuzzy random variable. We obviously well known that the trapezoidal fuzzy random variable curve is obtained from the symmetric triangular fuzzy random variable curve, when $\alpha=0.5=\frac{\alpha}{2}$.

The structure of the new trapezoidal fuzzy random variable is symmetric about its mean $\mu$. For that reason the new trapezoidal fuzzy random variable is now called symmetric trapezoidal fuzzy random variable and it is denoted by $\mathrm{X} \sim \operatorname{STPFRV}(\mu, \sigma)$

In this paper, we discuss about symmetric trapezoidal fuzzy random variable and its coordinate points $\left(\mu-\sigma, \mu-\frac{\sigma}{2}, \mu+\frac{\sigma}{2}, \mu+\sigma\right)$ are obtained from its parameters mean $\mu$, standard deviation $\sigma$ respectively.

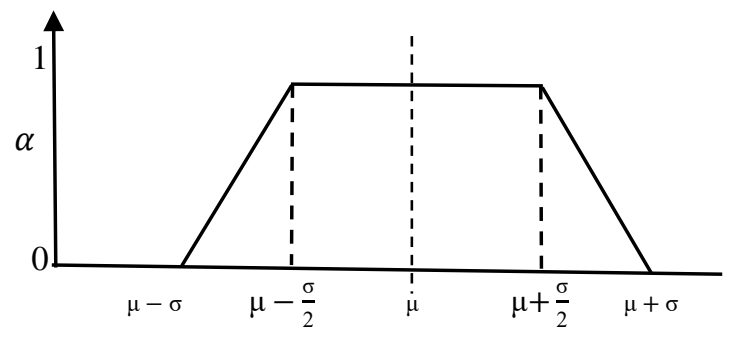

Figure 1. The symmetric trapezoidal fuzzy random variable

In our approach, we can find the finite sub - support for any $\alpha$ - cut value under the same membership function and two symmetric trapezoidal fuzzy random variables have same $\alpha$-cut value which are in the arithmetic operations. The organization of this paper is as follows. Section 2 is gives the basic definitions which are useful to build the symmetric trapezoidal fuzzy random variable with mean $\mu$ and standard deviation $\sigma$ respectively. In section 3, shows the relationship between symmetric triangular and symmetric trapezoidal fuzzy random variable and that we adopt our new definitions of symmetric trapezoidal fuzzy random variable. In section 4 , we derive the membership functions of the arithmetic operations between the two symmetric trapezoidal fuzzy random variables. In section 5 , we verify the membership function of the arithmetic operations of two symmetric trapezoidal fuzzy random variables through some numerical examples.

\section{PRELIMINARIES}

In this paper, we introduce a new concepts symmetric trapezoidal fuzzy random variable with mean $\mu$ and standard deviation $\sigma$. Under our approach, the co-ordinate points of continuous symmetric trapezoidal fuzzy random variable $X\left(\mu-\sigma, \mu-\frac{\sigma}{2}, \mu+\frac{\sigma}{2}, \mu+\sigma\right)$ are determined by its parameters mean $\mu$ and standard deviation $\sigma$ respectively. The membership function of $X$ can be written as

$\mathrm{P}\left\{\left(\mathrm{X}_{\alpha}^{\mathrm{L}}+\left(1-\frac{\alpha}{2}\right) \sigma-\mu\right) \geq 0 \mathrm{~V}\left(\mathrm{X}_{\alpha}^{\mathrm{U}}-\left(1-\frac{\alpha}{2}\right) \sigma-\mu\right) \leq 0\right\}$. 
Whole of this paper, we consider $X \sim \operatorname{STPFRV}\left(\mu_{1}, \sigma_{1}\right)$ and $Y \sim \operatorname{STPFRV}\left(\mu_{2}, \sigma_{2}\right)$ with conditions $(\mathrm{s} \leq X \leq \mathrm{t})$ and $(\mathrm{u} \leq Y \leq \mathrm{v})$. Here, $\mathrm{s}=\mu_{1}-\sigma_{1}, \mathrm{t}=\mu_{2}-\sigma_{2}, \mathrm{u}=\mu_{1}+\sigma_{1}$ and $\mathrm{v}$ $=\mu_{2}+\sigma_{2}$. The following definitions are used in succeeding sections.

\subsection{Definition}

A fuzzy set $\mathrm{A}$ is defined as

$$
\mathrm{A}=\left\{X, \mu_{\mathrm{A}}(\mathrm{x}): \mathrm{x} \in \mathrm{A}, \mu_{\mathrm{A}}(\mathrm{x}) \in[0,1]\right\}
$$

\subsection{Definition}

The support of fuzzy set A is the set of all points $x$ in $X$ such that $\mu_{A}(x)>0$. (i.e.,) $\operatorname{support}(A)=\left\{X / \mu_{A}(x)>0\right\}$.

\subsection{Definition}

The $\alpha$-cut of $\alpha$-level set of fuzzy set $A$ is a set consisting of those elements of the universe $X$ whose membership values exceed the threshold level $\alpha$.

(i.e.) $\mathrm{A}_{\alpha}=\left\{\mathrm{X} / \mu_{\mathrm{A}}(\mathrm{x}) \geq \alpha\right\}$

\subsection{Definition}

A fuzzy set A on $\mathrm{R}$ must possess at least the following three properties to qualify as a fuzzy number.

- A must be a normal fuzzy set.

- $\mathrm{A}_{\alpha}$ must be closed interval for every $\alpha \in[0,1]$.

- The support of $\mathrm{A},{ }^{0+} \mathrm{A}$ must be bounded.

Among the various shapes of fuzzy number, trapezoidal fuzzy number is the most popular one.

\subsection{Definition}

The symmetric trapezoidal fuzzy number is a fuzzy number represented with $4-$ tuples $A=\left(a_{1}, a_{2}, a_{3}, a_{4}\right)$. This representation is interpreted as membership function and holds the following conditions.

- $a_{1}$ to $a_{2}$ is increasing function

- $\mathrm{a}_{3}$ to $\mathrm{a}_{4}$ is decreasing function

- $\frac{a_{2}+a_{3}}{2}$ is mean (middle or symmetric point)

$$
\mu_{A}(X)=\left\{\begin{array}{cc}
0, & \text { for } X \leq a_{1} \\
\frac{x-a_{1}}{a_{2}-a_{1}}, & \text { for } a_{1} \leq X \leq a_{2} \\
1, & \text { for } a_{2} \leq X \leq a_{3} \\
\frac{a_{4}-x}{a_{4}-a_{3}}, & \text { for } a_{3} \leq X \leq a_{4} \\
0, & \text { for } a_{4} \geq X
\end{array}\right.
$$

Now, the $\alpha$-cut of the symmetric trapezoidal fuzzy number $\left[\mathrm{X}_{\alpha}^{\mathrm{L}}, \mathrm{X}_{\alpha}^{\mathrm{U}}\right]$ is

$\left(\mathrm{X}_{\alpha}^{\mathrm{L}}-\left(\mathrm{a}_{2}-\mathrm{a}_{1}\right) \alpha-\mathrm{a}_{1}\right) \geq 0, \quad\left(\mathrm{X}_{\alpha}^{\mathrm{U}}+\left(\mathrm{a}_{4}-\mathrm{a}_{3}\right) \alpha-\mathrm{a}_{3}\right) \leq 0$

Applying the values of

$A\left(a_{1}, a_{2}, a_{3}, a_{4}\right)=A\left(\mu_{1}-\sigma_{1}, \mu_{1}-\frac{\sigma_{1}}{2}, \mu_{1}+\frac{\sigma_{1}}{2}, \mu_{1}+\sigma_{1}\right)$

We get $\mathrm{X}_{\alpha}^{\mathrm{L}}-\left(\mathrm{a}_{2}-\mathrm{a}_{1}\right) \alpha-\mathrm{a}_{1}=\left(\mathrm{X}_{\alpha}^{\mathrm{L}}+\left(1-\frac{\alpha}{2}\right) \sigma-\mu\right) \geq 0$

$\mathrm{X}_{\alpha}^{\mathrm{U}}+\left(\mathrm{a}_{4}-\mathrm{a}_{3}\right) \alpha-\mathrm{a}_{3}=\left(\mathrm{X}_{\alpha}^{\mathrm{U}}-\left(1-\frac{\alpha}{2}\right) \sigma-\mu\right) \leq 0$
Therefore, the $\alpha$ - cut of the symmetric trapezoidal fuzzy random variable is

$$
\mathrm{P}\left\{\left(\mathrm{X}_{\alpha}^{\mathrm{L}}+\left(1-\frac{\alpha}{2}\right) \sigma-\mu\right) \geq 0 \vee\left(\mathrm{X}_{\alpha}^{\mathrm{U}}-\left(1-\frac{\alpha}{2}\right) \sigma-\mu\right) \leq 0\right\}
$$

\section{SYMMETRIC TRAPEZOIDAL FUZZY RANDOM VARIABLE}

\subsection{Relationship Between Symmetric Triangular And Symmetric Trapezoidal Fuzzy Random Variable}

\subsection{Theorem}

If $X$ be a symmetric triangular fuzzy random variable with mean $\mu_{1}$ and standard deviation $\sigma_{1}$ respectively and $\alpha=0.5$, then $X \sim \operatorname{STPFRV}\left(\mu_{1}, \sigma_{1}\right)$.

\section{Proof:}

The membership function of symmetric triangular fuzzy random variable $X \quad$ is $\mathrm{P}\left\{\left(\mathrm{X}_{\alpha}^{\mathrm{L}}+(1-\alpha) \sigma_{1}-\mu_{1}\right) \geq 0 \vee\left(\mathrm{X}_{\alpha}^{\mathrm{U}}-(1-\alpha) \sigma_{1}-\mu_{1}\right) \leq 0\right\}$ and placed $\alpha=0.5$, then the symmetric triangular shape become the symmetric trapezoidal shape with the same support, mean $\mu_{1}$ and standard deviation $\sigma_{1}$ respectively. So that the membership function of symmetric trapezoidal fuzzy random variable is obtained by put $\alpha=\frac{\alpha}{2}$ in the membership function of symmetric triangular fuzzy random variable, we get

$$
\mathrm{P}\left\{\left(\mathrm{X}_{\alpha}^{\mathrm{L}}+\left(1-\frac{\alpha}{2}\right) \sigma_{1}-\mu_{1}\right) \geq 0 \mathrm{~V}\left(\mathrm{X}_{\alpha}^{\mathrm{U}}-\left(1-\frac{\alpha}{2}\right) \sigma_{1}-\mu_{1}\right) \leq 0\right\}
$$

Which is the required symmetric trapezoidal fuzzy random variable with mean $\mu_{1}$ and standard deviation $\sigma_{1}$ respectively.

\section{Note:}

If $0<\alpha<0.5$ and $0.5<\alpha<1$, in the membership function of symmetric triangular fuzzy random variable, then we can also get the trapezoidal fuzzy random variable, but not symmetric.

\subsection{Membership Function Of Symmetric Trapezoidal Fuzzy Random Variable}

A fuzzy number $\mathrm{A}=(\mathrm{a}, \mathrm{b}, \mathrm{c}, \mathrm{d} ; \alpha)$ is said to be a symmetric trapezoidal fuzzy number and its membership function

$$
\mu_{A}(x)=\left\{\begin{array}{cc}
\frac{(x-a)}{(b-a)} & , \quad a \leq x<b \\
1 & , \quad b \leq x<c \\
\frac{(d-X)}{(d-c)} & , \quad, \quad x x<d
\end{array}\right.
$$

First, we derive the membership function of the lower $\alpha-$ cut of the symmetric trapezoidal fuzzy random variable is as follows: Applying the values of $\mathrm{A}$

$$
\begin{aligned}
\mathrm{A}(\mathrm{a}, \mathrm{b}, \mathrm{c}, \mathrm{d})= & \mathrm{A}\left(\mu-\sigma, \mu-\frac{\sigma}{2}, \mu+\frac{\sigma}{2}, \mu+\sigma\right) \\
& \frac{(\mathrm{x}-\mathrm{a})}{(\mathrm{b}-\mathrm{a})}=\frac{(\mathrm{x}-\mu+\sigma)}{\left(\mu-\frac{\sigma}{2}-\mu+\sigma\right)} \geq \alpha
\end{aligned}
$$




\section{E-ISSN: 2321-9637}

\section{Available online at www.ijrat.org}

$$
\left.\mathrm{X}_{a}^{\mathrm{L}}+\left(1-\frac{\alpha}{2}\right) \sigma-\mu\right) \geq 0
$$

Therefore, $\quad \mathrm{P}\left\{\left(\mathrm{X}_{\alpha}^{\mathrm{L}}+\left(1-\frac{\alpha}{2}\right) \sigma-\mu\right) \geq 0\right\}$

Which is the required lower $\alpha-$ cut of the symmetric trapezoidal fuzzy random variable.

Next, the membership function of the upper $\alpha$ - cut of the symmetric trapezoidal fuzzy random variable $X$ is derived as follows: Applying the values of $\mathrm{A}$

$$
\begin{aligned}
A(a, b, c, d)= & A\left(\mu-\sigma, \mu-\frac{\sigma}{2}, \mu+\frac{\sigma}{2}, \mu+\sigma\right) \\
& \frac{(d-X)}{(d-c)}=\frac{(\mu+\sigma-x)}{\left(\mu+\sigma-\mu-\frac{\sigma}{2}\right)} \geq \alpha \\
& \left.X_{\alpha}^{U}-\left(1-\frac{\alpha}{2}\right) \sigma-\mu\right) \leq 0 \\
\alpha &
\end{aligned}
$$

Therefore, $\mathrm{P}\left\{\left(\mathrm{X}_{\alpha}^{\mathrm{U}}-\left(1-\frac{\alpha}{2}\right) \sigma-\mu\right) \leq 0\right\}$

Which is the required upper $\alpha$ - cut of the symmetric trapezoidal fuzzy random variable.

Hence, the membership function of the symmetric trapezoidal fuzzy random variable $\mathrm{X}$ is

$$
\mathrm{P}\left\{\left(\mathrm{X}_{\alpha}^{\mathrm{L}}+\left(1-\frac{\alpha}{2}\right) \sigma-\mu\right) \geq 0 \mathrm{~V}\left(\mathrm{X}_{\alpha}^{\mathrm{U}}-\left(1-\frac{\alpha}{2}\right) \sigma-\mu\right) \leq 0\right\}
$$

\section{ARITHMETIC OPERATIONS OF SYMMETRIC TRAPEZOIDAL FUZZY RANDOM VARIABLES}

In this section, we consider $X \sim \operatorname{STPFRV}\left(\mu_{1}, \sigma_{1}\right)$, $Y \sim \operatorname{STPFRV}\left(\mu_{2}, \sigma_{2}\right)$ and its membership function is

$$
\begin{aligned}
& \mathrm{P}\left\{\left(\mathrm{X}_{\alpha}^{\mathrm{L}}+\left(1-\frac{\alpha}{2}\right) \sigma_{1}-\mu_{1}\right) \geq 0 \vee\left(\mathrm{X}_{\alpha}^{\mathrm{U}}-\left(1-\frac{\alpha}{2}\right) \sigma_{1}-\mu_{1}\right) \leq 0\right\} \\
& \mathrm{P}\left\{\left(\mathrm{Y}_{\alpha}^{\mathrm{L}}+\left(1-\frac{\alpha}{2}\right) \sigma_{2}-\mu_{2}\right) \geq 0 \vee\left(\mathrm{Y}_{\alpha}^{\mathrm{U}}-\left(1-\frac{\alpha}{2}\right) \sigma_{2}-\mu_{2} \leq 0\right\} .\right.
\end{aligned}
$$

\subsection{Addition Of Symmetric Trapezoidal Fuzzy Random Variables}

\subsection{Theorem}

Let $X \sim \operatorname{STPFRV}\left(\mu_{1}, \sigma_{1}\right)$ and $Y \sim \operatorname{STPFRV}\left(\mu_{2}, \sigma_{2}\right)$, then find the membership function of $((X+Y)) \sim \operatorname{STPFRV}\left(\mu_{1}+\mu_{2}, \sigma_{1}+\sigma_{2}\right)$.

\section{Proof:}

Mean $(X)=\mu_{1}$ and $\operatorname{Mean}(Y)=\mu_{2}$, then Mean $(X+$ $Y)=\mu_{1}+\mu_{2}$. According to J. Vahidi and S.Rezvani [7], the co - ordinate point of $(X+Y)$ is

$\left(\left(\mu_{1}+\mu_{2}\right)-\left(\sigma_{1}+\sigma_{2}\right),\left(\mu_{1}+\mu_{2}\right)-\frac{\left(\sigma_{1}+\sigma_{2}\right)}{2}, \mu_{1}+\mu_{2},\left(\mu_{1}+\mu_{2}\right)+\frac{\left(\sigma_{1}+\sigma_{2}\right)}{2}\right.$ , its membership function $\mu_{\mathrm{A}}(\mathrm{X}+\mathrm{Y})=$

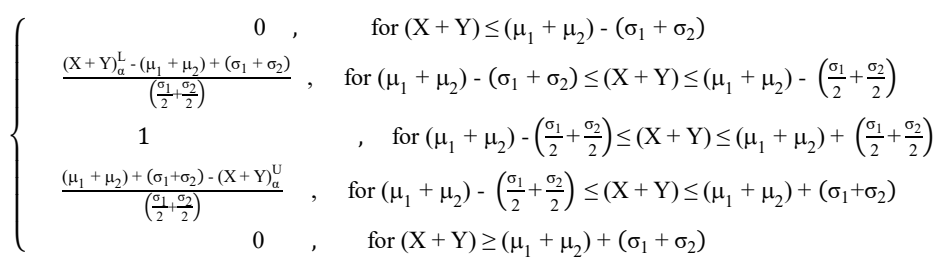

We obtain the lower and upper $\alpha$ - cut of symmetric trapezoidal fuzzy random variable $(X+Y)$ is given below:

\section{Derivation of lower limit}

$\frac{(\mathrm{X}+\mathrm{Y})_{\alpha}^{\mathrm{L}}-\left(\mu_{1}+\mu_{2}\right)+\left(\sigma_{1}+\sigma_{2}\right)}{\left(\frac{\sigma_{1}}{2}+\frac{\sigma_{2}}{2}\right)} \geq \alpha$

$(\mathrm{X}+\mathrm{Y})_{\alpha}^{\mathrm{L}}-\left(\mu_{1}+\mu_{2}\right) \geq\left(\sigma_{1}+\sigma_{2}\right)\left(\frac{\alpha}{2}-1\right)$

$\left((\mathrm{X}+\mathrm{Y})_{\alpha}^{\mathrm{L}}-\left(\mu_{1}+\mu_{2}\right)+\left(1-\frac{\alpha}{2}\right)\left(\sigma_{1}+\sigma_{2}\right)\right) \geq 0$

$\mathrm{P}\left\{(\mathrm{X}+\mathrm{Y})_{\alpha}^{\mathrm{L}}-\left(\mu_{1}+\mu_{2}\right)+\left(\sigma_{1}+\sigma_{2}\right)\left(1-\frac{\alpha}{2}\right) \geq 0\right\}$

Derivation of upper limit

$$
\begin{gathered}
\frac{\left(\mu_{1}+\mu_{2}\right)+\left(\sigma_{1}+\sigma_{2}\right)-(\mathrm{X}+\mathrm{Y})_{\alpha}^{\mathrm{U}}}{\left(\frac{\sigma_{1}}{2}+\frac{\sigma_{2}}{2}\right)} \geq \alpha \\
\left(\mu_{1}+\mu_{2}\right)-(\mathrm{X}+\mathrm{Y})_{\alpha}^{\mathrm{U}} \geq\left(\sigma_{1}+\sigma_{2}\right)\left(\frac{\alpha}{2}-1\right) \\
\left((\mathrm{X}+\mathrm{Y})_{\alpha}^{\mathrm{U}}-\left(\mu_{1}+\mu_{2}\right)-\left(\sigma_{1}+\sigma_{2}\right)\left(1-\frac{\alpha}{2}\right)\right) \leq 0 \\
\mathrm{P}\left\{\left((\mathrm{X}+\mathrm{Y})_{\alpha}^{\mathrm{U}}-\left(\mu_{1}+\mu_{2}\right)-\left(\sigma_{1}+\sigma_{2}\right)\left(1-\frac{\alpha}{2}\right)\right) \leq 0\right\}
\end{gathered}
$$

Hence, the membership function of sum of the symmetric quadratic triangular fuzzy random variable is given by

$$
\begin{gathered}
\mathrm{P}\left\{\left((\mathrm{X}+\mathrm{Y})_{\alpha}^{\mathrm{L}}-\left(\mu_{1}+\mu_{2}\right)+\left(\sigma_{1}+\sigma_{2}\right)\left(1-\frac{\alpha}{2}\right)\right) \geq 0\right. \\
\left.\mathrm{V}\left((\mathrm{X}+\mathrm{Y})_{\alpha}^{\mathrm{U}}-\left(\mu_{1}+\mu_{2}\right)-\left(\sigma_{1}+\sigma_{2}\right)\left(1-\frac{\alpha}{2}\right)\right) \leq 0\right\}
\end{gathered}
$$

The result is also a symmetric trapezoidal fuzzy random variables $(X+Y)$ with mean $\left(\mu_{1}+\mu_{2}\right)$ and standard deviation $\left(\sigma_{1}+\sigma_{2}\right)$ respectively.

\subsection{Subtraction of Symmetric Trapezoidal Fuzzy Random Variables}

\subsection{Theorem}

Let $X \sim \operatorname{STPFRV}\left(\mu_{1}, \sigma_{1}\right)$ and $Y \sim \operatorname{STPFRV}\left(\mu_{2}, \sigma_{2}\right)$, then find the membership function of $((X-Y)) \sim \operatorname{STPFRV}\left(\mu_{1}-\mu_{2}, \sigma_{1}+\sigma_{2}\right)$.

\section{Proof:}

Mean $(X)=\mu_{1}$ and $\operatorname{Mean}(Y)=\mu_{2}$, then Mean $(X-$ $Y)=\mu_{1}-\mu_{2}$. According to J. Vahidi and S.Rezvani [7], the co - ordinate point of $(\mathrm{X}-\mathrm{Y})$ is

$\left(\left(\mu_{1}-\mu_{2}\right)-\left(\sigma_{1}+\sigma_{2}\right),\left(\mu_{1}-\mu_{2}\right)-\frac{\left(\sigma_{1}+\sigma_{2}\right)}{2}, \mu_{1}-\mu_{2},\left(\mu_{1}-\right.\right.$ $\left.\left.\mu_{2}\right)+\frac{\left(\sigma_{1}+\sigma_{2}\right)}{2},\left(\mu_{1}-\mu_{2}\right)+\left(\sigma_{1}+\sigma_{2}\right)\right)$, its membership function $\mu_{\mathrm{A}}(\mathrm{X}-\mathrm{Y})=$

$$
\begin{aligned}
& \left(0, \quad \text { for }(X-Y) \leq\left(\mu_{1}-\mu_{2}\right)-\left(\sigma_{1}+\sigma_{2}\right)\right. \\
& \frac{(X-Y)_{a}^{L}-\left(\mu_{1}-\mu_{2}\right)+\left(\sigma_{1}+\sigma_{2}\right)}{\left(\frac{\sigma_{1}}{2}+\frac{\sigma_{2}}{2}\right)} \text {, for }\left(\mu_{1}-\mu_{2}\right)-\left(\sigma_{1}+\sigma_{2}\right) \leq(X-Y) \leq\left(\mu_{1}-\mu_{2}\right)-\left(\frac{\sigma_{1}}{2}+\frac{\sigma_{2}}{2}\right) \\
& 1 \quad \text {, for }\left(\mu_{1}-\mu_{2}\right)-\left(\frac{\sigma_{1}}{2}+\frac{\sigma_{2}}{2}\right) \leq(X-Y) \leq\left(\mu_{1}-\mu_{2}\right)+\left(\frac{\sigma_{1}}{2}+\frac{\sigma_{2}}{2}\right) \\
& \frac{\left(\mu_{1}+\mu_{2}\right)+\left(\sigma_{1}+\sigma_{2}\right)-(X-Y)_{a}^{U}}{\left(\sigma_{1} \sigma_{2}\right)} \text {, for }\left(\mu_{1}-\mu_{2}\right)-\left(\frac{\sigma_{1}}{2}+\frac{\sigma_{2}}{2}\right) \leq(X-Y) \leq\left(\mu_{1}-\mu_{2}\right)+\left(\sigma_{1}+\sigma_{2}\right)
\end{aligned}
$$

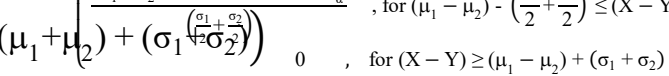

We obtain the lower and upper $\alpha$ - cut of symmetric trapezoidal fuzzy random variable $(\mathrm{X}-\mathrm{Y})$ is given below:

\section{Derivation of lower limit}

$$
\begin{aligned}
& \frac{(X-Y)_{\alpha}^{L}-\left(\mu_{1}-\mu_{2}\right)+\left(\sigma_{1}+\sigma_{2}\right)}{\left(\frac{\sigma_{1}}{2}+\frac{\sigma_{2}}{2}\right)} \geq \alpha \\
& (X-Y)_{\alpha}^{L}-\left(\mu_{1}-\mu_{2}\right) \geq\left(\sigma_{1}+\sigma_{2}\right)\left(\frac{\alpha}{2}-1\right) \\
& \left((X-Y)_{\alpha}^{L}-\left(\mu_{1}-\mu_{2}\right)+\left(1-\frac{\alpha}{2}\right)\left(\sigma_{1}+\sigma_{2}\right)\right) \geq 0
\end{aligned}
$$




\section{E-ISSN: 2321-9637}

\section{Available online at www.ijrat.org}

$\mathrm{P}\left\{(\mathrm{X}-\mathrm{Y})_{\alpha}^{\mathrm{L}}-\left(\mu_{1}-\mu_{2}\right)+\left(\sigma_{1}+\sigma_{2}\right)\left(1-\frac{\alpha}{2}\right) \geq 0\right\}$

Derivation of upper limit

$$
\frac{\left(\mu_{1}-\mu_{2}\right)+\left(\sigma_{1}+\sigma_{2}\right)-(X-Y)_{\alpha}^{U}}{\left(\frac{\sigma_{1}}{2}+\frac{\sigma_{2}}{2}\right)} \geq \alpha
$$

$\left(\mu_{1}-\mu_{2}\right)-(X-Y)_{\alpha}^{U} \geq\left(\sigma_{1}+\sigma_{2}\right)\left(\frac{\alpha}{2}-1\right)$

$\left((X-Y)_{\alpha}^{U}-\left(\mu_{1}-\mu_{2}\right)-\left(\sigma_{1}+\sigma_{2}\right)\left(1-\frac{\alpha}{2}\right)\right) \leq 0$

$\mathrm{P}\left\{\left((\mathrm{X}-\mathrm{Y})_{\alpha}^{\mathrm{U}}-\left(\mu_{1}-\mu_{2}\right)-\left(\sigma_{1}+\sigma_{2}\right)\left(1-\frac{\alpha}{2}\right)\right) \leq 0\right\}$

Hence, the membership function of sum of the symmetric quadratic triangular fuzzy random variable is given by

$$
\begin{array}{r}
\mathrm{P}\left\{\left((\mathrm{X}-\mathrm{Y})_{\alpha}^{\mathrm{L}}+\left(1-\frac{\alpha}{2}\right)\left(\sigma_{1}+\sigma_{2}\right)-\left(\mu_{1}-\mu_{2}\right)\right) \geq 0\right. \\
\mathrm{V}\left((\mathrm{X}-\mathrm{Y})_{\alpha}^{\mathrm{U}}-\left(1-\frac{\alpha}{2}\right)\left(\sigma_{1}+\sigma_{2}\right)-\left(\mu_{1}-\mu_{2}\right)\right) \leq 0
\end{array}
$$

The result is also a symmetric trapezoidal fuzzy random variables $(\mathrm{X}-\mathrm{Y})$ with mean $\left(\mu_{1}-\mu_{2}\right)$ and standard deviation $\left(\sigma_{1}+\sigma_{2}\right)$ respectively.

\subsection{Multiplication Of Symmetric Trapezoidal Fuzzy Random Variables}

\subsection{Theorem}

Let $X \sim \operatorname{STPFRV}\left(\mu_{1}, \sigma_{1}\right)$ and $Y \sim \operatorname{STPFRV}\left(\mu_{2}, \sigma_{2}\right)$, then derive the membership function of $((X \cdot Y)) \sim \operatorname{TPFRV}\left(\mu_{1} \mu_{2}\right)$, but not symmetric.

\section{Proof:}

Mean $(X)=\mu_{1}$ and $\operatorname{Mean}(Y)=\mu_{2}$, then Mean $(X \cdot Y)=\mu_{1} \mu_{2}$. According to Vahidi. J, Rezvani. S [7], the coordinate points of multiplication of two symmetrical trapezoidal fuzzy random variables is $\left(\left(\mu_{1}-\sigma_{1}\right)\left(\mu_{2}-\sigma_{2}\right),\left(\mu_{1}-\frac{\sigma_{1}}{2}\right)\left(\mu_{2}-\frac{\sigma_{2}}{2}\right),\left(\mu_{1} \mu_{2}\right),\left(\mu_{1}+\frac{\sigma_{1}}{2}\right)\left(\mu_{2}+\frac{\sigma_{2}}{2}\right),\left(\mu_{1}+\sigma_{1}\right)\left(\mu_{2}\right.\right.$ $\left.+\sigma_{2}\right)$, ),

$$
\text { its } \mu_{\mathrm{A}}(\mathrm{X} \cdot \mathrm{Y})=\left\{\begin{array}{cc}
\text { membership } & \text { function } \\
0 & \text { for } 0 \leq(X \cdot Y) \leq\left(\mu_{1}-\sigma_{1}\right)\left(\mu_{2}+\sigma_{2}\right) \\
\frac{(\mathrm{X} \cdot \mathrm{Y})-\left(\mu_{1}-\sigma_{1}\right)\left(\mu_{2}+\sigma_{2}\right)}{\left(\mu_{1}-\frac{\sigma_{1}}{2}\right)\left(\mu_{2}-\frac{-2}{2}\right)-\left(\mu_{1}-\sigma_{1}\right)\left(\mu_{2}+\sigma_{2}\right)}, & \text { for }\left(\mu_{1}-\sigma_{1}\right)\left(\mu_{2}+\sigma_{2}\right) \leq(X \cdot Y) \leq\left(\mu_{1}-\frac{\sigma_{1}}{2}\right)\left(\mu_{2}-\frac{\sigma_{2}}{2}\right) \\
1, & \text { for }\left(\mu_{1}-\frac{\sigma_{1}}{2}\right)\left(\mu_{2}-\frac{\sigma_{2}}{2}\right) \leq(X \cdot Y) \leq\left(\mu_{1}+\frac{\sigma_{1}}{2}\right)\left(\mu_{2}+\frac{\sigma_{2}}{2}\right) \\
\frac{\left(\mu_{1}+\sigma_{1}\right)\left(\mu_{2}+\sigma_{2}\right)-(X \cdot Y)}{\left(\mu_{1}+\sigma_{1}\right)\left(\mu_{2}+\sigma_{2}\right)-\left(\mu_{1}+\frac{\sigma_{1}}{2}\right)\left(\mu_{2}+\frac{\sigma_{2}}{2}\right)}, & \text { for }\left(\mu_{1}+\frac{\sigma_{1}}{2}\right)\left(\mu_{2}+\frac{\sigma_{2}}{2}\right) \leq(X \cdot Y) \leq\left(\mu_{1}+\sigma_{1}\right)\left(\mu_{2}+\sigma_{2}\right) \\
0, & \text { for }(X \cdot Y) \geq\left(\mu_{1}+\sigma_{1}\right)\left(\mu_{2}+\sigma_{2}\right)
\end{array}\right.
$$

First, we obtain the lower $\alpha$ - cut of symmetric trapezoidal fuzzy random variable $(X \cdot Y)$ is given below:

$$
\frac{(\mathrm{X} \cdot \mathrm{Y})_{\alpha}^{\mathrm{L}}-\left(\mu_{1}-\sigma_{1}\right)\left(\mu_{2}-\sigma_{2}\right)}{\left(\mu_{1}-\frac{\sigma_{1}}{2}\right)\left(\mu_{2}-\frac{\sigma_{2}}{2}\right)-\left(\mu_{2}-\sigma_{2}\right)\left(\mu_{1}-\sigma_{1}\right)} \geq \alpha
$$

On simplification, we get

$$
\begin{aligned}
& \left.(X \cdot Y)_{\alpha}^{\mathrm{L}}-\mu_{1} \sigma_{2}\left(\frac{\alpha}{2}-1\right)-\mu_{2} \sigma_{1}\left(\frac{\alpha}{2}-1\right)-\sigma_{1} \sigma_{2}\left(1-\frac{3 \alpha}{4}\right)-\mu_{1} \mu_{2}\right) \geq 0 \\
& \left.\quad \mathrm{P}\left\{(\mathrm{X} \cdot \mathrm{Y})_{\alpha}^{\mathrm{L}}-\mu_{1} \sigma_{2}\left(\frac{\alpha}{2}-1\right)-\mu_{2} \sigma_{1}\left(\frac{\alpha}{2}-1\right)-\sigma_{1} \sigma_{2}\left(1-\frac{3 \alpha}{4}\right)-\mu_{1} \mu_{2}\right) \geq 0\right\}
\end{aligned}
$$

Which is the required lower $\alpha$-cut of the multiplication of symmetric trapezoidal fuzzy random variable.

Secondly, we derive the upper $\alpha$ - cut of symmetric trapezoidal fuzzy random variable $(X \cdot Y)$.

$$
\begin{gathered}
\frac{\left(\mu_{1}+\sigma_{1}\right)\left(\mu_{2}-\sigma_{2}\right)-(X \cdot Y)_{\alpha}^{\mathrm{U}}}{\left(\mu_{1}+\sigma_{1}\right)\left(\mu_{2}-\sigma_{2}\right)-\left(\mu_{1}+\frac{\sigma_{1}}{2}\right)\left(\mu_{2}+\frac{\sigma_{2}}{2}\right)} \geq \alpha \\
\frac{\left(\mu_{1}+\sigma_{1}\right)\left(\mu_{2}+\sigma_{2}\right)-(\mathrm{X} \cdot \mathrm{Y})_{\alpha}^{\mathrm{U}}}{\left(\frac{\mu_{1} \sigma_{2}}{2}+\frac{\mu_{2} \sigma_{1}}{2}+\frac{3 \sigma_{1} \sigma_{2}}{4}\right)} \geq \alpha \\
\left.(\mathrm{X} \cdot \mathrm{Y})_{\alpha}^{\mathrm{U}}+\mu_{1} \sigma_{2}\left(\frac{\alpha}{2}-1\right)+\mu_{2} \sigma_{1}\left(\frac{\alpha}{2}-1\right)+\sigma_{1} \sigma_{2}\left(\frac{3 \alpha}{4}-1\right)-\mu_{1} \mu_{2}\right) \leq 0 \\
\mathrm{P}\left\{\left((\mathrm{X} \cdot \mathrm{Y})_{\alpha}^{\mathrm{U}}+\mu_{1} \sigma_{2}\left(\frac{\alpha}{2}-1\right)+\mu_{2} \sigma_{1}\left(\frac{\alpha}{2}-1\right)+\sigma_{1} \sigma_{2}\left(\frac{3 \alpha}{4}-\right.\right.\right. \\
\left.\left.1)-\mu_{1} \mu_{2}\right) \leq 0\right\}
\end{gathered}
$$

Which is the required upper $\alpha$ - cut of the symmetric trapezoidal fuzzy random variable.

Therefore, the membership function of multiplication of two symmetric trapezoidal fuzzy random variable $(X \cdot Y)$ is

$\mathrm{P}\left\{\left((\mathrm{X} \cdot \mathrm{Y})_{\alpha}^{\mathrm{L}}-\mu_{1} \sigma_{2}\left(\frac{\alpha}{2}-1\right)-\mu_{2} \sigma_{1}\left(\frac{\alpha}{2}-1\right)-\sigma_{1} \sigma_{2}\left(1-\frac{3 \alpha}{4}\right)-\mu_{1} \mu_{2}\right) \geq\right.$
$\left.\mathrm{V}\left((\mathrm{X} \cdot \mathrm{Y})_{\alpha}^{\mathrm{U}}+\mu_{1} \sigma_{2}\left(\frac{\alpha}{2}-1\right)+\mu_{2} \sigma_{1}\left(\frac{\alpha}{2}-1\right)+\sigma_{1} \sigma_{2}\left(\frac{3 \alpha}{4}-1\right)-\mu_{1} \mu_{2}\right) \leq 0\right\}$

The result of multiplication of two symmetric trapezoidal fuzzy random variable is also a trapezoidal fuzzy random variable, but it is not symmetric.

\subsection{Division Of Symmetric Trapezoidal Fuzzy Random Variables}

\subsection{Theorem}

Let $X \sim \operatorname{STPFRV}\left(\mu_{1}, \sigma_{1}\right)$ and $Y \sim \operatorname{STPFRV}\left(\mu_{2}, \sigma_{2}\right)$, then obtain the membership function of $((X / Y)) \sim \operatorname{TPFRV}\left(\mu_{1} /\right.$ $\mu_{2}$ ), but not symmetric.

\section{Proof:}

Mean $(X)=\mu_{1}$ and Mean $(Y)=\mu_{2}$, then $\operatorname{Mean}(X / Y)=\mu_{1} /$ $\mu_{2}$. According to Vahidi. J, Rezvani. S [7], the coordinate points of division of two symmetric trapezoidal fuzzy random variables is

$(X / Y)=\left(\frac{\mu_{1}-\sigma_{1}}{\mu_{2}+\sigma_{2}}, \frac{\mu_{1}-\frac{\sigma_{1}}{2}}{\mu_{2}+\frac{\sigma_{2}}{2}}, \frac{\mu_{1}}{\mu_{2}}, \frac{\mu_{1}+\frac{\sigma_{1}}{2}}{\mu_{2}-\frac{\sigma_{2}}{2}}, \frac{\mu_{1}+\sigma_{1}}{\mu_{2}-\sigma_{2}}\right), \quad$ and its membership function is

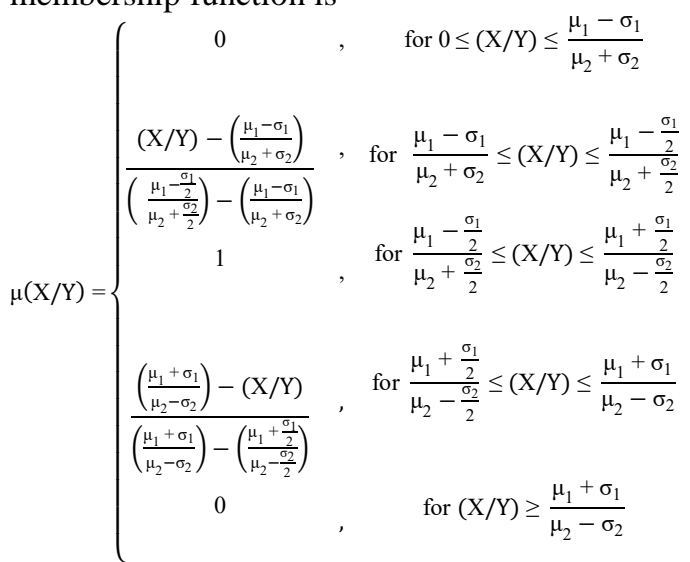

First, we derive the lower $\alpha$-cut of symmetric trapezoidal fuzzy random variables $(X / Y)$ is as follows 


$$
\frac{(\mathrm{X} / \mathrm{Y})-\frac{\mu_{1}-\sigma_{1}}{\mu_{2}+\sigma_{2}}}{\frac{\left(\mu_{1}-\frac{\sigma_{1}}{2}\right)\left(\mu_{2}+\sigma_{2}\right)-\left(\mu_{1}-\sigma_{1}\right)\left(\mu_{2}+\frac{\sigma_{2}}{2}\right)}{\left(\mu_{2}+\frac{\sigma_{2}}{2}\right)\left(\mu_{2}+\sigma_{2}\right)}} \geq \alpha
$$

On simplification we get,

$$
\begin{aligned}
& (X / Y) \geq \frac{\left(\mu_{1} \sigma_{2}(\alpha+1)+\mu_{2} \sigma_{1}(\alpha-2)+2 \mu_{1} \mu_{2}-\sigma_{1} \sigma_{2}\right)}{\left(2 \mu_{2}+\sigma_{2}\right)\left(\mu_{2}+\sigma_{2}\right)} \\
& P\left\{\left((X / Y)_{\alpha}^{\mathrm{L}}\right.\right. \\
& \left.-\frac{\left(\mu_{1} \sigma_{2}(\alpha+1)+\mu_{2} \sigma_{1}(\alpha-2)+2 \mu_{1} \mu_{2}-\sigma_{1} \sigma_{2}\right)}{\left(2 \mu_{2}+\sigma_{2}\right)\left(\mu_{2}+\sigma_{2}\right)} \geq 0\right\}
\end{aligned}
$$

Which is the required lower $\alpha$ - cut of the symmetric trapezoidal fuzzy random variables.

Secondly, we obtain the upper $\alpha$-cut of symmetric trapezoidal fuzzy random variables $(X / Y)$ is as follows

$$
\frac{\frac{\mu_{1}+\sigma_{1}}{\mu_{2}-\sigma_{2}}-(X / Y)}{\frac{\left(\mu_{2}-\frac{\sigma_{2}}{2}\right)\left(\mu_{1}+\sigma_{1}\right)-\left(\mu_{2}-\sigma_{2}\right)\left(\mu_{1}+\frac{\sigma_{1}}{2}\right)}{\left(\mu_{2}-\frac{\sigma_{2}}{2}\right)\left(\mu_{2}-\sigma_{2}\right)}} \geq \alpha
$$

On simplification, we get

$$
\begin{array}{r}
(\mathrm{X} / \mathrm{Y}) \leq-\frac{\left(\mu_{1} \sigma_{2}(\alpha+1)+\mu_{2} \sigma_{1}(\alpha-2)-2 \mu_{1} \mu_{2}+\sigma_{1} \sigma_{2}\right)}{\left(2 \mu_{2}-\sigma_{2}\right)\left(\mu_{2}-\sigma_{2}\right)} \\
\mathrm{P}\left\{\left(\left((\mathrm{X} / \mathrm{Y})_{\alpha}^{\mathrm{U}}+\frac{\left(\mu_{1} \sigma_{2}(\alpha+1)+\mu_{2} \sigma_{1}(\alpha-2)-2 \mu_{1} \mu_{2}+\sigma_{1} \sigma_{2}\right)}{\left(2 \mu_{2}-\sigma_{2}\right)\left(\mu_{2}-\sigma_{2}\right)} \leq 0\right\}\right.\right.
\end{array}
$$

Which is the required upper $\alpha$ - cut of the symmetric trapezoidal fuzzy random variables.

Therefore, the membership function of division of two symmetric trapezoidal fuzzy random variables $(X / Y)$ is $\mathrm{P}\left\{\left((\mathrm{X} / \mathrm{Y})_{\alpha}^{\mathrm{L}}\right.\right.$

$$
\begin{aligned}
& -\frac{\left(\mu_{1} \sigma_{2}(\alpha+1)+\mu_{2} \sigma_{1}(\alpha-2)+2 \mu_{1} \mu_{2}-\sigma_{1} \sigma_{2}\right)}{\left(2 \mu_{2}+\sigma_{2}\right)\left(\mu_{2}+\sigma_{2}\right)} \geq 0 \\
& V\left((X / Y)_{\alpha}^{\mathrm{U}}+\frac{\left(\mu_{1} \sigma_{2}(\alpha+1)+\mu_{2} \sigma_{1}(\alpha-2)-2 \mu_{1} \mu_{2}+\sigma_{1} \sigma_{2}\right)}{\left(2 \mu_{2}-\sigma_{2}\right)\left(\mu_{2}-\sigma_{2}\right)} \leq 0\right\}
\end{aligned}
$$

\begin{tabular}{|c|c|c|c|c|c|c|c|c|}
\hline \multicolumn{2}{|l|}{$X$} & \multicolumn{2}{|l|}{$Y$} & \multirow{2}{*}{$\begin{array}{l}\text { Operations } \\
\text { / Equation }\end{array}$} & \multirow{2}{*}{$\begin{array}{c}\mu=0 \\
\left(\mathrm{X}_{\alpha}^{\mathrm{L}}, \mathrm{X}_{\alpha}^{\mathrm{U}}\right)\end{array}$} & \multirow{2}{*}{$\begin{array}{c}\mu=1 \\
\left(\mathrm{X}_{\alpha}^{\mathrm{L}}, \mathrm{X}_{\alpha}^{\mathrm{U}}\right)\end{array}$} & \multirow{2}{*}{$\begin{array}{l}\text { Co - ordinate } \\
\text { point }\end{array}$} & \multirow{2}{*}{$\begin{array}{l}\text { Mean, } \\
\text { Standard } \\
\text { deviation }\end{array}$} \\
\hline$\mu_{1}$ & $\sigma_{1}$ & $\mu_{2}$ & $\sigma_{2}$ & & & & & \\
\hline 3 & 1 & 6 & 1 & $\begin{array}{r}\text { Addition / } \\
\text { (4.1) }\end{array}$ & $(7,11)$ & $(8,10)$ & $(7,8,10,11)$ & $\begin{array}{r}\mu_{1}+\mu_{2}=9, \\
\sigma_{1}+\sigma_{2}=2\end{array}$ \\
\hline 4 & 5 & 3 & 1 & $\begin{array}{l}\text { Subtractio } \\
\mathrm{n} / \quad(4.2)\end{array}$ & $(-0.5,2.5)$ & $\begin{array}{l}(0.25 \\
1.75) \\
\end{array}$ & $\begin{array}{r}(-0.5,0.25 \\
1.75,2.5) \\
\end{array}$ & $\begin{array}{c}\mu_{1}-\mu_{2}=1, \\
\sigma_{1}+\sigma_{2}=1.5\end{array}$ \\
\hline 3 & 2 & 4 & 2 & $\begin{array}{l}\text { Multiplicat } \\
\text { ion/ } \quad(4.3)\end{array}$ & $(2,30)$ & $(6,20)$ & $(2,6,20,30)$ & $\begin{array}{l}\mu_{1} \cdot \mu_{2}=12 \\
\text { Asymmetric }\end{array}$ \\
\hline 3 & 2 & 5 & 2 & $\begin{array}{r}\text { Division / } \\
\text { (4.4) }\end{array}$ & $\left(\frac{1}{7}, \frac{5}{3}\right)$ & $\left(\frac{1}{3}, 1\right)$ & $\left(\frac{1}{7}, \frac{1}{3}, 1, \frac{5}{3}\right)$ & $\begin{array}{c}\frac{\mu_{1}}{\mu_{2}}=\frac{3}{5} \\
\text { Asymmetric }\end{array}$ \\
\hline
\end{tabular}

The result of division of two symmetric trapezoidal fuzzy random variable is trapezoidal fuzzy random variable, but it is not symmetric.

\section{NUMERICAL EXAMPLES}

\subsection{Example}

The following table shows that the arithmetic operations between symmetric trapezoidal fuzzy random variables by using various values of its parameters.

\section{REFERENCE}

[1] Féron. R, "Ensembles aleatoires flous", C.R. Acad. Sci. Paris, 282, PP: 903-906, 1976.

[2] Kwakernaak. H., "Fuzzy random variables - I, Definitions and theorems", Information Science, 15, PP: 1-29, 1978.

[3] Kwakernaak. H., "Fuzzy random variables - II, Algorithms and Examples for the Discrete Case", Information Science, 17(3), PP: 253 - 278, (1979).

[4] Puri. M.L and Ralescu, D.A., "Fuzzy random variables", Journal of Mathematical Analysis and Applications, 114, PP: 409 - 422, 1986.

[5] Puri. M. L and Ralescu. D. A., "The concept of normality for fuzzy random variables", Ann Probab., 13(4), PP: 1373 -1379, 1985.

[6] Rajan.D and Senthil Murugan. C, “A New Approach on Orderings of Triangular Fuzzy Random Variables", International Journal of Fuzzy Mathematical Archive, Vol. 9(2), 153 - 163, 2015.

[7] Vahidi. J, Rezvani. S, "Arithmetic operations on trapezoidal fuzzy numbers", Journal Nonlinear Analysis and Application, 2013, 1 - 8, 2013.

[8] Zadeh, L.A., "Fuzzy sets", Information and Control, 8, PP: 338 - 353, 1965. 